\title{
Cooperative Spectrum Sensing
}

\author{
Jinlong Wang, Qihui Wu, Xueqiang Zheng \\ and Juan Chen \\ Institute of Communications Engineering \\ PLA University of Science and Technology
}

China

\section{Introduction}

With the rapid growth of wireless applications and services in the recent decade, spectrum resources are facing huge demands. Traditionally, the frequency spectrum is licensed to users by government agencies in a rigid manner where the licensee has the exclusive right to access the allocated band. Recent measurements by Spectrum Policy Task Force (SPTF) within the FCC indicate that, the allocated spectrums are vastly underutilized sporadically and geographically with a high variance in time (McHenry, 2005). There are increasing interests in increasing the spectrum efficiency. The exciting findings shed light on the problem of spectrum scarcity and motivate a new direction to solve the conflicts between spectrum scarcity and spectrum under-utilization.

Cognitive radio (CR) enables much higher spectrum efficiency by dynamic spectrum access (Mitola \& Maguire, 1999). Therefore, it is a potential technique for future wireless communications to mitigate the spectrum scarcity issue. CR implementations face many technical challenges, including spectrum sensing, dynamic frequency selection, adaptive modulation, and wideband frequency-agile RF front-end circuitry. These challenges are compounded by the inherent transmission impairments of wireless links, user mobility, non uniform legacy radio resource allocation policies, and user dependent economic considerations.

Obviously, spectrum sensing is a critical functionality of CR networks, it allows cognitive users to detect spectral holes and to opportunistically use under-utilized frequency bands without causing harmful interference to legacy systems.

In cognitive radio system, when cognitive users are sensing the channel, the sampled received signal of cognitive users has two hypotheses. Hypothesis $H_{1}$ denotes the primary user is active, and hypothesis $H_{0}$ denotes the primary user is inactive.

$$
\begin{aligned}
& H_{1}: y(k)=h * s(k)+n(k) \\
& H_{0}: y(k)=n(k)
\end{aligned}
$$


where $y(k)$ is the signal received by cognitive user, $s(k)$ is the transmitted signal of the primary user. The signal $s(k)$ is distorted by the channel gain $h$, which is assumed to be constant during the detection interval, and is further corrupted by the zero-mean additive white Gaussian noise $n(k)$ with the variance $\sigma_{n}^{2}$. Without loss of generality, $s(k)$ and $n(k)$ are assumed to be independent of each other. $r=|h|^{2} E_{s} / \sigma_{n}{ }^{2}$ denotes the signal-tonoise ratio, where $E_{s}$ is the signal energy of the primary user.

Given a single frequency band, the first challenge for $C R$ is to reliably detect the existence of primary user to minimize the interference to existing communications. A number of different methods are proposed for identifying the presence of signal transmission, such as matched filter detection, energy detection, feature detection techniques and wavelet approach (Arslan \& Yucek, 2007).

However, the hidden terminal problem occurs when cognitive user is shadowed, in severe multipath fading or inside buildings with high penetration loss while primary user is operating in the vicinity. Due to the hidden terminal problem, the sensing performance for one cognitive user will be degraded.

To prevent the hidden terminal problem, the CR network could fuse the sensing results of multiple cognitive users and exploit spatial diversity among distributed cognitive users to enhance the sensing reliability.

In this way, a network of spatially distributed cognitive users, which experience different channel conditions from the target, would have a better chance of detecting the primary user by exchanging sensing information. Therefore, collaborative spectrum sensing can alleviate the problem of corrupted detection by exploiting the built-in spatial diversity to reduce the probability of interfering with primary users.

Since collaborative sensing is generally coordinated over a reporting channel, efficient cooperation schemes should be investigated to reduce bandwidth and power requirements while optimizing the sensing reliability. Important design considerations include the overhead reduction associated with sensing information exchange and the feasibility issue of reporting channels. In general, operating characteristics (such as false alarm versus detection probabilities) of the detector should be selected by considering the achievable opportunistic throughput of cognitive users and the probability of no colliding with primary user.

\section{Classification of Cooperative Spectrum Sensing}

Cooperative sensing can be implemented in two fashions: centralized or distributed (Akyildiz et al, 2006). These two fashions will be explained in the following sections.

\subsection{Centralized Sensing}

In centralized sensing, a fusion centre collects sensing information from cognitive users. The fusion centre identifies the available spectrum, and broadcasts the fused result to other cognitive users or directly controls cognitive user's traffic. According to whether cognitive users exchange sensing information themselves, the centralized sensing fashion can also be divided to two categories. 


\section{A Partially Cooperative Network}

Each of CR users detects the spectrum independently and directly transmits its sensing information to the fusion centre.

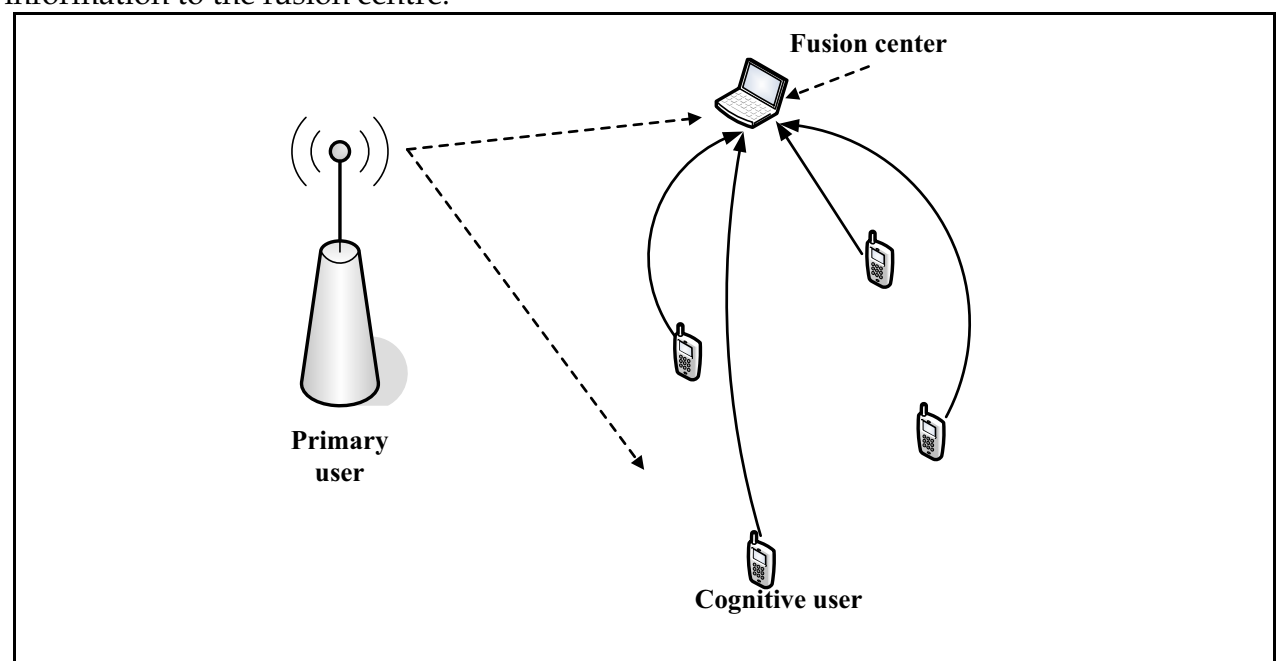

Fig. 1. Scheme of the partially cooperative network

\section{B Totally Cooperative Network}

Cognitive users cooperatively transmit each others sensing information, and then send sensing information to the fusion centre.

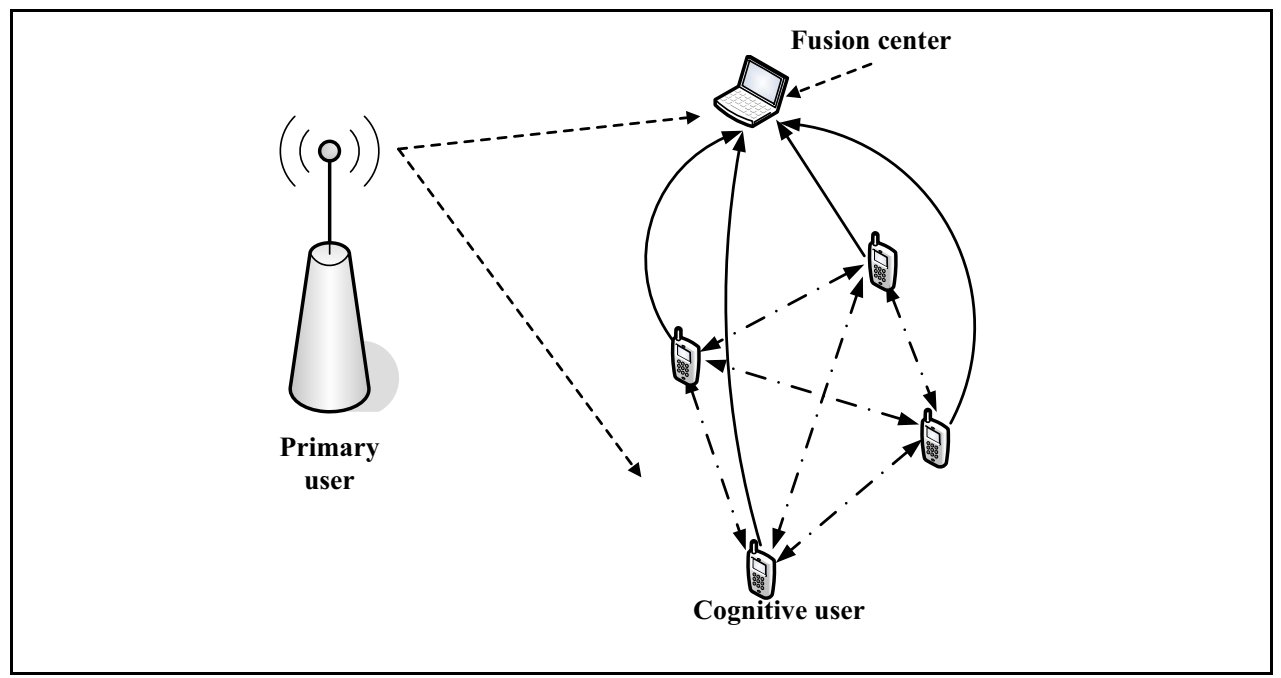

Fig. 2. Scheme of the totally cooperative network 


\subsection{Distributed Sensing}

In the case of distributed sensing, cognitive users share information among each other but they make their own decisions as to which part of the spectrum they can use. Distributed sensing is more advantageous in the sense that there is no need for a backbone infrastructure.

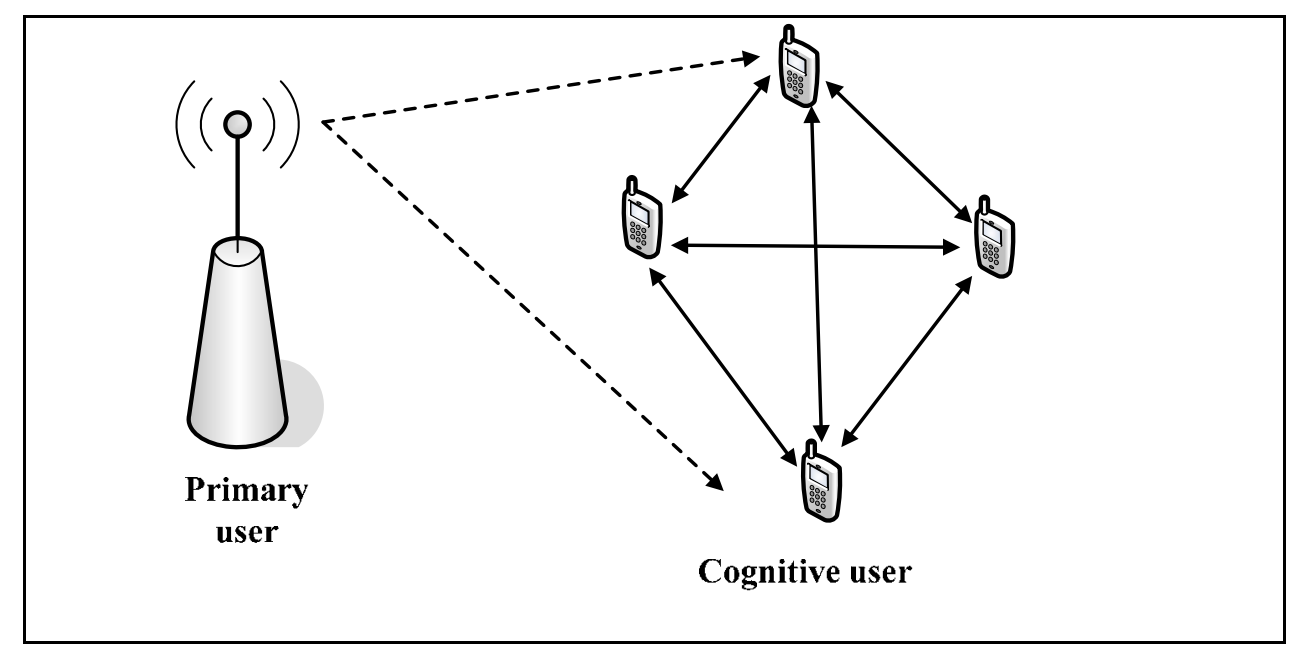

Fig. 3. Scheme of the distributed sensing

We focus on centralized sensing in the following sections.

\section{The Fusion Rules}

The cognitive users collaborate by reporting their local observations to the fusion centre, which makes the final decision on whether $H_{1}$ or $H_{0}$ is true. Due to bandwidth and other practical constraints, the cognitive users may report only quantized observations. Here, two classes of fusion algorithms, hard information-combining and soft information-combining algorithm, are considered. In the former, each cognitive user $i$ performs a local hypothesis test and reports a binary variable $\mathrm{B}_{\mathrm{i}}=1$ if it believes $H_{1}$ is true, and $\mathrm{B}_{\mathrm{i}}=0$ otherwise. In the latter, each user reports the full observation to the fusion centre. The sensing performance of cooperative spectrum sensing is related to the local observations and the fusion rules.

\subsection{Hard-decision Fusion Rules}

One of the simplest suboptimal solutions to the data fusion problem is the Counting Rule (Aalo \& Viswanathan, 1992) (also referred to as the Voting Rule), which counts the number of cognitive users that vote for the presence of the signal and compares it against a given threshold. Equivalently, the decision is based solely on the type of the received vector of bits. Based on voting rules, in (Ghasemi \& Sousa, 2005), a fusion rule known as the OR logic operation was used to combine decisions from several cognitive users, the sensing performance is given as follows: 


$$
\left\{\begin{array}{l}
P_{O R_{-} D}=1-\prod_{i=1}^{N}\left(1-P_{D i}\right) \\
P_{O R_{-} F}=1-\prod_{i=1}^{N}\left(1-P_{F i}\right)
\end{array}\right.
$$

where $\mathrm{N}$ is the number of cognitive users, P_Di and P_Fi are the probability of the detection and false alarm.

In (Visotsky et al, 2005), hard decision with the AND logic operation was proposed, the sensing performance is given as follows,

$$
\left\{\begin{array}{l}
P_{A N D_{-} D}=\prod_{i=1}^{N} P_{D i} \\
P_{A N D_{-} F}=\prod_{i=1}^{N} P_{F i}
\end{array}\right.
$$

A general suboptimal solution to the fusion problem was given in (Unnikrishnan \& Veeravalli, 2008) when the signal received by cognitive users are correlated. This solution uses partial statistical knowledge and gives better performance than the one obtained by ignoring the correlation information completely.

An optimum data fusion structure has been derived in (Chair \& Varshney, 1986) which combines the decisions from the individual detectors while minimizing the overall probability of error. Individual decisions are weighted according to their reliability, that is, the weights are a function of the probability of detection and false alarm of the individual user. The fusion and decision rule is as follow.

$$
\alpha_{0}+\sum_{i=1}^{N} \alpha_{i} u_{i} \stackrel{H_{1}}{<} 0
$$

where $u_{i}$ is the local decision of the $i$ th cognitive user,the weighting factor $\alpha_{i}$ is defined as follows.

$$
\alpha_{i}= \begin{cases}\log \frac{P_{1}}{P_{0}}, & i=0 \\ \log \frac{P_{D i}}{P_{F i}}, & u_{i}=+1 \\ \log \frac{1-P_{F i}}{1-P_{D i}}, & u_{i}=-1\end{cases}
$$




\subsection{Soft-decision Fusion Rules}

If the channel which cognitive users report the sensing results to the fusion centre is not limited by bandwidth, each cognitive user can report sensing information without any compression. Then the performance of collaborative spectrum sensing will be greatly enhanced. In (Visotsky et al., 2005), both soft information-combining strategy and hard information-combining strategy are introduced to detect the primary user. It was shown that the soft information-combining strategy has better detecting performance.

When cognitive users did not know any prior knowledge of the primary user, energy fusion method is the optimal fusion of the detection statistics. The test statistic for energy detector is $\theta=\sum_{k=1}^{2 u}|y(k)|^{2}$, where $u$ is the time-bandwidth product. Given an instantaneous $r, \theta$ follows the distribution ( Visotsky et al., 2007).

$$
f(\theta \mid r) \sim\left\{\begin{array}{rr}
x_{2 u}^{2} & H_{0} \\
x_{2 u}^{2}(2 r) & H_{1}
\end{array}\right.
$$

where $r$ is exponentially distributed with the mean value $\bar{r}, u$ is the time-bandwidth product of the energy detector, $x_{2 u}^{2}$ represents a central chi-square distribution with $2 u$ degrees of freedom, and $x_{2 u}^{2}(2 r)$ represents a non-central chi-square distribution with $2 u$ degrees of freedom and a non-centrality parameter $2 r$.

If cognitive users know the prior knowledge of the primary users, soft decision using the likelihood ratio test (LRT) is optimal. The LRT-based optimal fusion rule (Lim et al., 2008) involves a quadratic form as a result of high computational complexity. The closed expressions of detection probability and false alarm probability can not be derived.

The optimal linear framework for cooperative spectrum sensing in cognitive radio networks is proposed in (Quan et al., 2008). The proposed methods optimize the detection performance by operating over a linear combination of local test statistics from individual cognitive users, which combats the destructive channel effects between the target primary user and the opportunistic cognitive users.

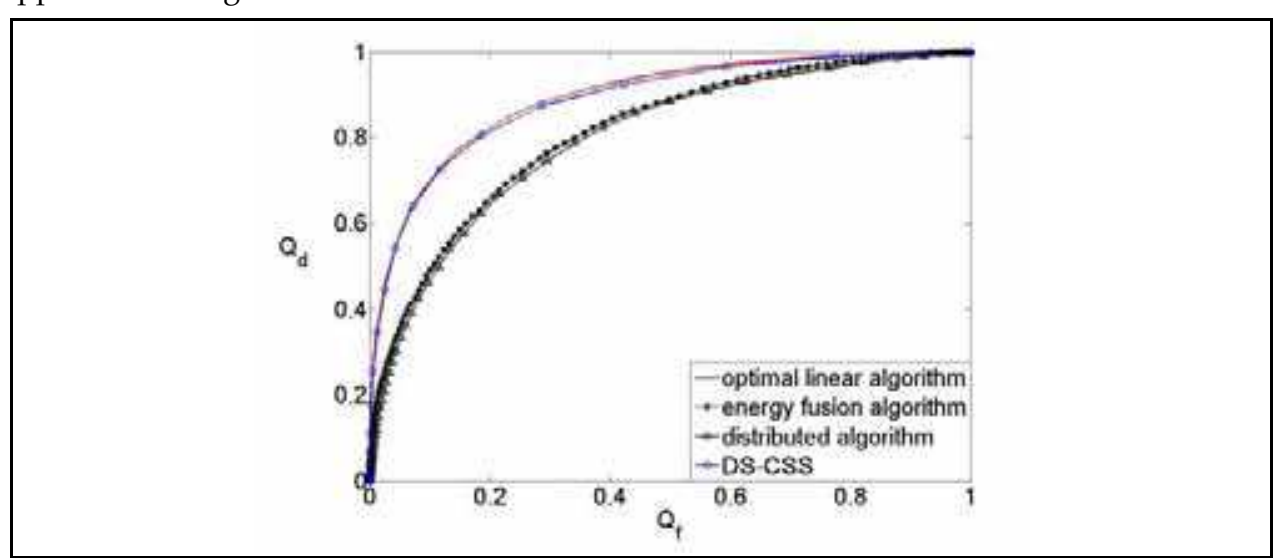

Fig. 4. ROC of DS-CSS 
The optimal linear detection provides performance comparable to that achievable by the optimal LRT-based fusion in many situations. But the fusion centre needs to know the prior knowledge of the primary user and all cognitive users.

In order to efficiently detect the licensed users under prior knowledge constraints in the fusion centre, a novel cooperative spectrum sensing algorithm based on Dempster-Shafer theory (DS-CSS) is proposed in (Zheng et al, 2008). In this novel algorithm, the credibility and uncertainty are calculated based on the local sensing result of cognitive users, then fused at the fusion centre using Dempster-Shafer theory. Fig.4 shows that the performance of the novel algorithm is better than energy fusion method.

\section{Network Overhead Reduction}

Except the sensing performance, network overhead for cooperation is also important for the cooperative spectrum sensing. At present, there are mainly two ways to reduce the network overhead. One is to reduce the sensing information which cognitive users send to the fusion centre. Another is to reduce the number of reporting cognitive radios.

\subsection{Compression of Sensing information}

Usually, the first way to reduce the network overhead is to reduce the amount of information which each cognitive user transmits through the reporting channel, that is, the results of the local perception compression to achieve the purpose of network overhead reduction.

At present most of the hard-information combination algorithms fall into this category, such as the AND criteria, OR criteria $\mathrm{K} / \mathrm{M}$ criteria and so on.

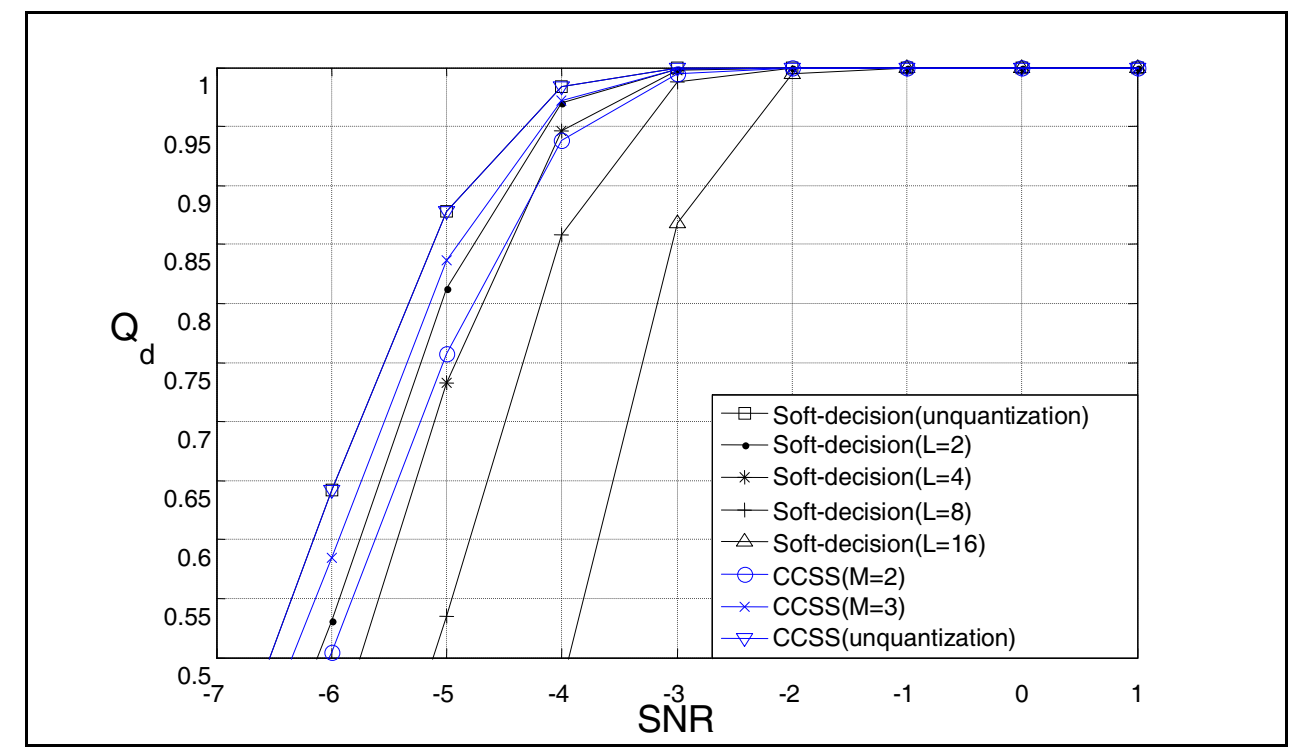

Fig. 5. The sensing performance of CCSS algorithm 
Quantization of the local sensing information is another way to reduce the amount of the reporting information. The quantization in signal detection problems were studied in (Hashlamoun \& Varshney, 1996) (Helstrom, 1988) (Chamberland \& Veeravalli, 2004)( Blum, 1999). The influence of the number of quantization bits were studied in (Kaligineedi et al., 2008) ( Taherpour et al., 2007). A new softened hard combination scheme with two-bit overhead for each user is proposed in (Ma et al., 2008), and this scheme can achieve a good tradeoff between sensing performance and complexity.

To reduce the network overhead of soft information-combining strategy with little expense of performance loss, a novel credible cooperative spectrum sensing (CCSS) algorithm was given in (Zheng et al., 2009). We considered the potential of using the reliability of cognitive users for cooperative spectrum sensing in cognitive radio systems. Analysis showed that it was possible to achieve high probabilities of detection as soft information-combining strategy by proper fusion of the cognitive users' decisions. In particular, the close-form expressions for probabilities of detection and false-alarm were derived for novel algorithm, and expression for average overhead used for cooperation was given. The threshold designing method for the algorithm was also discussed. Fig.5 and Fig.6 show that the novel algorithm can perform as well as soft information-combining strategy and its overhead was much less than soft information-combining strategy ( $W$ in Fig. 6).

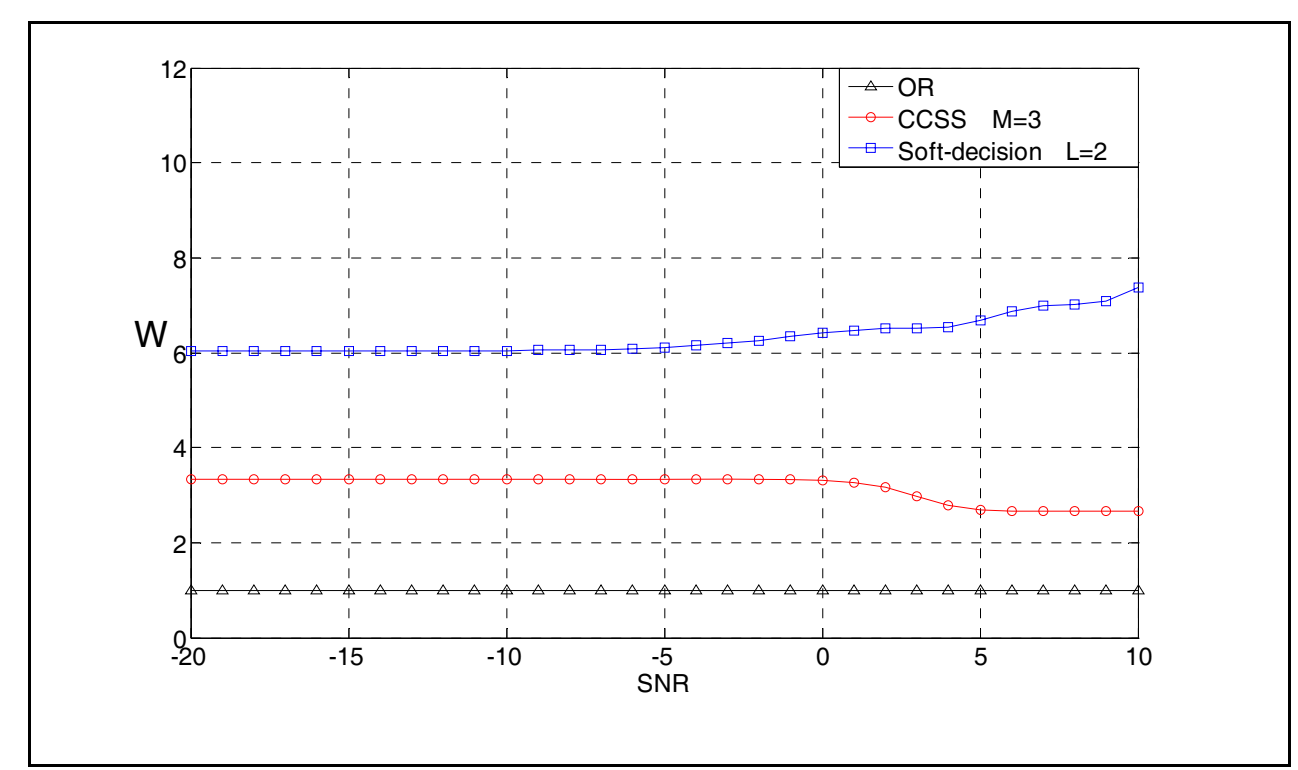

Fig. 6. The overhead of CCSS algorithm

\section{2 sensor selection}

Except compressing the sensing information of cognitive users, another method of network overhead reduction for cooperative spectrum sensing is to reduce the number of reporting cognitive users. For hard and soft information-combining algorithms, the sensor selection methods are different. 


\section{A Hard information-combining algorithms}

Firstly, for hard information-combining algorithm, there are some topics about it. In (Edward \& Liang, 2007), when cooperative spectrum sensing algorithms use AND and OR fusion schemes with energy detector, the optimum probabilities of detection and false alarm can be achieved by cooperating a certain number of users with the highest primary user's signal to noise ratio rather than cooperating all the users within the network. In (Zhang et al., 2008), the performance of cooperative spectrum sensing in cognitive radio networks have been studied. It has been found that the optimal decision fusion rule to minimize the total error probability is the half-voting rule. Moreover, the optimal detection threshold of energy detection is determined numerically. In particular, an efficient spectrum sensing algorithm has been proposed which requires fewer than the total number of cognitive radios in cooperative spectrum sensing while satisfying a given error bound.

In (Sun et al., 2008), a novel sensing user selection scheme is proposed. The optimal data fusion rule is used to simulate the sensing performance before and after the sensing nodes selection. Analysis and simulation results suggest that this method can reduce the number of sensing users remarkably and effectively without degrading the performance of sensing system obviously. Compared to the censoring method with quantization, this scheme can also reduce the performance deterioration when there are many cognitive users experience the same shadowing effects.

\section{B Soft information-combining algorithms}

Generally, when the fusion rule is soft information-combining rule, the sensing performance is best when all cognitive user in cognitive radio system participate cooperative spectrum sensing. However, the network overhead is the most in that situation. Considering the sensing performance and network overhead, the optimum number of cognitive users in collaborative spectrum sensing is derived in (Chen, 2008) for lognormal shadowing channels, static additive white Gaussian noise channels and Rayleigh fading channels, when the efficiency of resources usage is considered in the system design.

When there are $\mathrm{N}$ cognitive users, the network efficiency is

$$
\eta(n)=\frac{N-n}{N}
$$

The optimal number of cognitive radios is defined by weighting the sensing performance and the network efficiency in a target function

$$
J(n)=(1-\alpha) \cdot P_{d}(n)+\alpha \cdot \eta(n)
$$

where $J(n)$ is the target function, $\alpha$ is the impact factor, $P_{d}(n)$ is the probability of detection, where $n$ is the number of cognitive users participate in cooperative sensing.

$$
P_{d}(n)=P\left(H_{0}\right)\left(1-Q_{f}\right)+P\left(H_{1}\right)\left(1-Q_{m}\right)
$$

$Q_{f}$ and $Q_{m}$ are the probability of false alarm and miss detection respectively. 


$$
\left\{\begin{array}{l}
Q_{f}=P\left(H_{1} \mid H_{0}\right) \\
Q_{m}=P\left(H_{0} \mid H_{1}\right)
\end{array}\right.
$$

\section{Sensing Information Transmission}

Most present researches on cooperative spectrum sensing are based on the following assumptions: the reporting channel between the fusion centre and cognitive users is an ideal error-free transmission channel. But sometimes it is very difficult in practice to achieve the above-mentioned transmission conditions, the cognitive user's distance from the fusion centre may be far away, or there are obstacles in the transmission block, then the sensing information transmission are likely to be wrong.

When there exist reporting errors, the upper and lower limits of the false alarm probability was given in (Sun et al., 2007), but the solutions were not given. When the fusion rules are AND and OR logical operation, the probability of false alarm and miss detection are

$$
\begin{gathered}
\left\{\begin{array}{l}
Q_{f}^{O R}=1-\prod_{i=1}^{N}\left[\left(1-P_{f, i}\right)\left(1-P_{e, i}\right)+P_{f, i} P_{e, i}\right] \\
Q_{m}^{O R}=\prod_{i=1}^{N}\left[P_{m, i}\left(1-P_{e, i}\right)+\left(1-P_{m, i}\right) P_{e, i}\right]
\end{array}\right. \\
\left\{\begin{array}{l}
Q_{f}^{A N D}=\prod_{i=1}^{N}\left[\left(1-P_{f, i}\right) P_{e, i}+P_{f, i}\left(1-P_{e, i}\right)\right] \\
Q_{m}^{A N D}=1-\prod_{i=1}^{N}\left[\left(1-P_{m, i}\right)\left(1-P_{e, i}\right)+P_{m, i} P_{e, i}\right]
\end{array}\right.
\end{gathered}
$$

Suppose that the local spectrum sensing conducted by cognitive user $i$ results in $P_{f, i}=P_{f}$ and $P_{m, i}=P_{m}$, for all $i=1,2, \ldots, K$, and that the probabilities of reporting errors are identical for all CRs $P_{e, i}=P_{e}$, then the probability of false alarm and miss detection can be bounded by

$$
\begin{gathered}
Q_{f}^{O R} \geq \bar{Q}_{f} \triangleq \lim _{P_{f} \rightarrow 0} Q_{f}=1-\left(1-P_{e}\right)^{N} \approx N P_{e} \\
Q_{m}^{A N D} \geq \bar{Q}_{m} \triangleq \lim _{P_{m} \rightarrow 0} Q_{m}=1-\left(1-P_{e}\right)^{N} \approx N P_{e}
\end{gathered}
$$

It has been shown that using multiple cognitive users may improve the detection probability over realistic sensing and reporting channels. But the performance is limited by the probability of reporting error $P_{e}$ which is due to imperfect reporting channels.

In (Ganesan \& Li, 2008), two schemes have been analyzed considering sensing and reporting at the same time. The schemes employ the Amplify-and-forward (AF) cooperation protocol to reduce the detection time. It was shown that cooperation between cognitive users increases the overall agility of the network. 
Cooperative spectrum sensing taking into account the Decode-and-forward (DF) cooperation protocol on the decision reporting is studied in (Zhang \& Letaief, 2008). A transmit diversity method is given which applies some existing space-time (ST) coding and space-frequency (SF) coding for multiple antennas systems to the CR network by viewing cognitive users as distributed antenna arrays. It was found that cooperative spectrum sensing with DF cooperation protocol could increase the detection probability with reporting errors.

Space-time coding can be used for cooperative spectrum sensing but the performance is constrained because the information exchange between cognitive users may not be correctly performed. Network coding (NC) admits a larger rate region than routing at polynomial complexity. We propose a novel cooperative spectrum sensing algorithm based on network coding (NC-CSS) by considering the use of network coding for such information exchanges. The simulation results show that the performance of the NC-CSS algorithm is better than the above cooperative spectrum sensing algorithm based on space-time coding (ST-CSS), and the novel algorithm can meet the situation when there exist destroyed reporting paths. When there exists one destroyed reporting path, cooperative spectrum sensing with network coding can achieve better sensing performance, as in Fig.7.

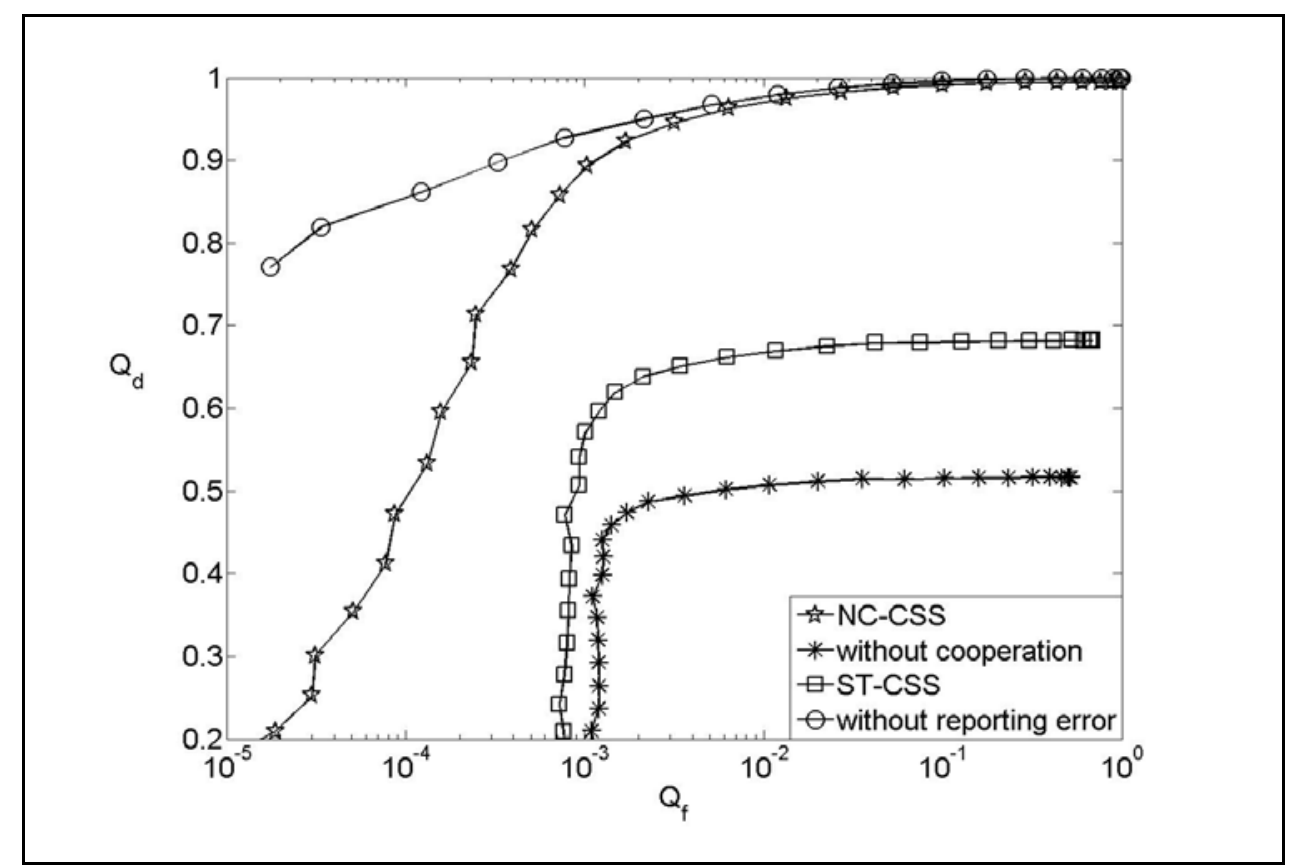

Fig. 7. ROC of NC-CSS algorithm

At present, there are still many issues unresolved in non-ideal reporting channel conditions, how to consider the synergy of the design spectrum sensing algorithm is particularly important. 


\section{Conclusion}

With the increasing demand for radio spectrum on one hand and inefficient usage of the licensed bands on the other, cognitive radio can efficiently utilize the spectrum holes of the licensed channels in different locations and times. To detect the spectrum holes accurately and quickly, spectrum sensing is a critical component in cognitive radio systems. By allowing a number of cognitive users to perform local spectrum sensing independently and fusing their local decision results together at the fusion centre, the spectrum sensing performance can be greatly enhanced.

In this chapter we investigate the main issues associated with the design of cooperative spectrum sensing functionality for cognitive radio system. Sensing performance is decided by the local decisions and fusion rules in the fusion centre. The network overhead for cooperation is also important for cooperative spectrum sensing, a good cooperative spectrum sensing algorithm should have better sensing performance with less network overhead. Cooperative spectrum sensing algorithms using space-time coding and network coding are also discussed when the reporting channel is imperfect.

An important venue for further research is the design of cooperative spectrum sensing considering the positions and mobility of cognitive users. Further research on cooperative spectrum sensing can be envisioned on wideband sensing.

\section{References}

Aalo V \& Viswanathan R.(1992) Asymptotic performance of a distributed detection system in correlated Gaussian noise, IEEE TRANSACTIONS ON SIGNAL PROCESSING, Vol. 40, pp. 211-213, 1992.

Akyildiz I F, Lee W Y, Vuran M C \& Mohanty S.(2006) Next generation/dynamic spectrum access/cognitive radio wireless networks:a survey, ElSEVIER COMPUTER NETWORKS JOURNAL, Vol. 50, No. 13, pp. 2127-2159.

Arslan H \& Yucek T.(2007) Spectrum Sensing for Cognitive Radio Applications, Cognitive Radio, Software Defined Radio, and Adaptive Wireless Systems, pp.263-289, Springer.

Blum R. (1999) Distributed Detection for Diversity Reception of Fading Signals in Noise, IEEE TRANSACTIONS ON INFORMATION THEORY, Vol. 45, No. 1, pp.158-164.

Chair Z \& Varshney P K.(1986) Optimal Data Fusion in Multiple Sensor Detection Systems. IEEE TRANSACTIONS ON AEROSPACE AND ELECTRONIC SYSTEMS, Vol. AES-22, No. 98,pp. 98-101.

Chamberland J \& Veeravalli V. (2004) The impact of fading on decentralized detection in power constrained wireless sensor networks, ICASSP 2004,pp.837-840.

Chen Y.(2008) Optimum Number of Secondary Users in Collaborative Spectrum Sensing Considering Resources Usage Efficiency. IEEE COMMUNICATIONS LETTERS, Vol. 12, No. 12, pp.877-879.

Digham F.F, Alouini M.S, and Simon M.K.(2007) On the Energy Detection of Unknown Signals Over Fading Channels, IEEE TRANSACTIONS ON COMMUNICATIONS, Vol.50, No.1, pp. 21-24.

Edward P \& Liang Y C.(2007) Optimization for Cooperative Sensing in Cognitive Radio Networks, WCNC 2007, pp.27-32. 
Ganesan G \& Li Y.(2007) Cooperative Spectrum Sensing in Cognitive Radio, Part I: Two User Networks[J]. IEEE TRANSACTIONS ON WIRELESS COMMUNICATIONS, Vol.6, No.6, pp. 2204-2213.

Taherpour A, Nasiri-Kenari M, Jamshidi A.(2007) Efficient Cooperative Spectrum Sensing in Cognitive Radio Networks, PIMRC2007.

Ghasemi A \& Sousa E.(2005) Collaborative Spectrum Sensing for Opportunistic Access in Fading Environments[C]. IEEE DySPAN 2005, pages: 131 - 136.(Ghasemi \& Sousa, 2005)

Hashlamoun W. A \& Varshney P K.(1999) Near-Optimum Quantization for Signal detection[J]. IEEE TRANSACTIONS ON COMMUNICATIONS, Vol. 44, No. 3, pp.294-297.

Helstrom C. (1988) Improved Multilevel Quantization for Detection of Narrowband Signals[J]. IEEE TRANSACTIONS ON AEROSFACE AND ELECTRONIC SYSTEMS , Vol. 24, No. 2, Pp.141-147.

Kaligineedi P, Khabbazian M, Bhargava V.(2008) Secure Cooperative Sensing Techniques for Cognitive Radio Systems. ICC 2008.

Lim T J, Zhang R, Liang Y C \& Zeng Y. (2008) GLRT-Based Spectrum Sensing for Cognitive Radio.GLOBECOM 2008.

Ma J, Zhao G \& Li Y.(2008) Soft Combination and Detection for Cooperative Spectrum Sensing in Cognitive Radio Networks. IEEE TRANSACTIONS ON WIRELESS COMMUNICATIONS, Vol. 7, No. 11, pp.4502-4507.

McHenry M A.(2005) NSF Spectrum Occupancy Measurements Project Summary, shared spectrum co. report.

Mitola J \& Maguire Q.(1999) Cognitive radio: Making software radios more personal, IEEE PERSONAL COMMUNICATIONS, Vol. 6, No. 4, pp. 13-18.

Quan Z, Cui S \& Sayed A H. (2008) Optimal Linear Cooperation for Spectrum Sensing in Cognitive radio Networks. IEEE JOURNAL OF SELECTED TOPICS IN SIGNAL PROCESSING, Vol. 2, No.1, pp.28-40.

Selen Y, Tullberg H, Kronander J.(2008) Sensor Selection for Cooperative Spectrum Sensing. DYSPAN 2008, pages: 1-11.

Sun C, Zhang W \& Letaief K B.(2007) Cluster-Based Cooperative Spectrum Sensing in Cognitive Radio Systems. ICC 2007, pp.2511-2515.

Sun Y, Hu H, Liu F et al.(2008) Selection of Sensing Nodes in Cognitive Radio System Based on Correlation of Sensing Information. CROWNCOM 2008.

Unnikrishnan J \& Veeravalli V.(2008) Cooperative Sensing for Primary Detection in Cognitive Radio. IEEE JOURNAL OF SELECTED TOPICS IN SIGNAL PROCESSING, Vol. 2, No. 1, pp.18-27.

Visotsky E, Kuffner S \& Peterson R.(2005) On collaborative detection of TV transmissions in support of dynamic spectrum sensing, Proc. IEEE DYSPAN, USA, pp. 338-345.

Zhang W \& Letaief K B.(2008) Cooperative Spectrum Sensing with Transmit and Relay Diversity in Cognitive Radio Networks[J]. IEEE TRANSACTIONS ON WIRELESS COMMUNICATIONS, Vol.7, No.12, pp. 4761-4766.

Zhang W, Mallik R K \& Letaief K B.(2008) Cooperative Spectrum Sensing Optimization in Cognitive Radio Networks. ICC 2008.

Zheng X, Wang J, Wu Q \& Chen J.(2008) Cooperative Spectrum Sensing Algorithm based on Dempster-Shafer Theory, ICCS 2008, pp.218-221. 
Zheng X, Wang J, Wu Q \& Shen L. (2009) A Novel Cooperative Spectrum Sensing Algorithm in Cognitive Radio Systems, JOURNAL OF COMMUNICATIONS AND NETWORKS, Vol.11,No.2,Apirl,2009,pp:115-121. (Zheng et al., 2009) 


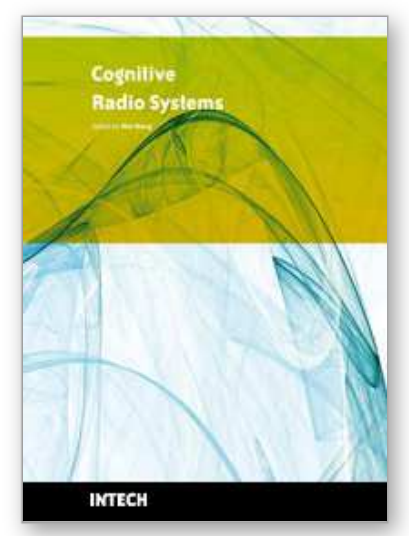

\section{Cognitive Radio Systems}

Edited by Wei Wang

ISBN 978-953-307-021-6

Hard cover, 340 pages

Publisher InTech

Published online 01, November, 2009

Published in print edition November, 2009

Cognitive radio is a hot research area for future wireless communications in the recent years. In order to increase the spectrum utilization, cognitive radio makes it possible for unlicensed users to access the spectrum unoccupied by licensed users. Cognitive radio let the equipments more intelligent to communicate with each other in a spectrum-aware manner and provide a new approach for the co-existence of multiple wireless systems. The goal of this book is to provide highlights of the current research topics in the field of cognitive radio systems. The book consists of 17 chapters, addressing various problems in cognitive radio systems.

\section{How to reference}

In order to correctly reference this scholarly work, feel free to copy and paste the following:

Jinlong Wang, Qihui Wu, Xueqiang Zheng and Juan Chen (2009). Cooperative Spectrum Sensing, Cognitive Radio Systems, Wei Wang (Ed.), ISBN: 978-953-307-021-6, InTech, Available from:

http://www.intechopen.com/books/cognitive-radio-systems/cooperative-spectrum-sensing

\section{INTECH}

open science | open minds

\section{InTech Europe}

University Campus STeP Ri Slavka Krautzeka 83/A

51000 Rijeka, Croatia

Phone: +385 (51) 770447

Fax: +385 (51) 686166

www.intechopen.com

\section{InTech China}

Unit 405, Office Block, Hotel Equatorial Shanghai No.65, Yan An Road (West), Shanghai, 200040, China 中国上海市延安西路65号上海国际贵都大饭店办公楼 405 单元

Phone: +86-21-62489820

Fax: +86-21-62489821 
(C) 2009 The Author(s). Licensee IntechOpen. This chapter is distributed under the terms of the Creative Commons Attribution-NonCommercial-ShareAlike-3.0 License, which permits use, distribution and reproduction for non-commercial purposes, provided the original is properly cited and derivative works building on this content are distributed under the same license. 